\title{
El laboratorio en la enseñanza de la fisiología: Narraciones de los estudiantes de Medicina Veterinaria
}

The laboratory in physiology teaching:

Tales of Veterinary Medicine students

\section{David Fernando Balaguera Quinche*}

(iD) : https://orcid.org/0000-0001-6857-7517

\section{Leonardo Gómez Duarte**}

(iD) : https://orcid.org/0000-0002-2623-1187

Doi de artículo: 10.17533/udea.unipluri.18.2.04

Cómo citar este artículo:

Balaguera, D. y Gómez, L. (2018). El Laboratorio en la enseñanza de la fisiología: narraciones de los estudiantes de Medicina Veterinaria. Uni-pluriversidad, 18(2), 57-69. https://doi.org/10.17533/udea. unipluri.18.2.04

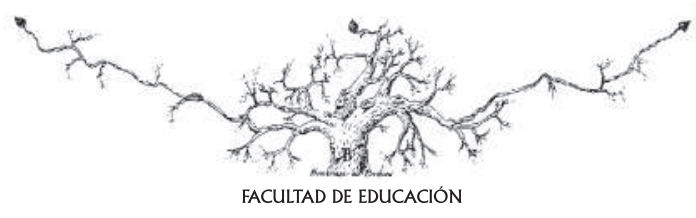

Recibido: 2018-10-08 • Aprobado: 2019-02-12

* Laboratorio de Fisiología y Farmacología, Facultad de Medicina Veterinaria y Zootecnia, Universidad Nacional de Colombia, Bogotá. Contacto: dfbalagueraq@unal.edu.co

** Departamento de Ciencias Fisiológicas, Facultad de Medicina, Universidad Nacional de Colombia, Bogotá. Contacto: olgomezd@gmail.com 


\title{
Resumen
}

Con el fin de explorar la visión de los estudiantes de Medicina Veterinaria de la Universidad Nacional de Colombia sobre el papel del laboratorio en la enseñanza de la fisiología se construyeron diarios de campo en donde los alumnos de relataron de manera personal los eventos vividos durante las prácticas con la metodología de aprendizaje basada en la indagación. El objetivo fue explorar la experiencia de los estudiantes mediante el análisis narrativo de algunos diarios con el fin de reconstruir una historia por cada estudiante durante su paso por el laboratorio. Como resultado se evidenció que para los estudiantes el laboratorio de fisiología puede ser un espacio para reforzar, poner en práctica y perpetuar el conocimiento, para la interacción social, la reflexión moral, un lugar fuera de la rutina, el desarrollo de habilidades de pensamiento superior y la intriga por explorar la naturaleza del organismo.

Palabras clave: laboratorio, fisiología, experiencia, diario, relatos

\begin{abstract}
In order to explore the vision of the students of Veterinary Medicine of the National University on the role of the laboratory in the teaching of physiology, field journals were constructed where the students related in a personal way the events experienced during the practices of laboratory with the methodology of learning based on inquiry. The objective was to explore the students' experience through the narrative analysis of some journals in order to reconstruct a story for each student during their passage through the laboratory. As a result it was evidenced that for students the physiology laboratory can be a space to reinforce, put into practice and perpetuate knowledge, for social interaction, moral reflection, a place outside of routine, the development of higher thinking skills and the intrigue to explore the nature of the organism.
\end{abstract}

Key words: laboratory, physiology, experience, diary, stories 


\section{Introdução}

El laboratorio como estrategia didáctica se ha usado en la enseñanza de la fisiología desde el comienzo de la disciplina y aun con más fuerza desde su cambio de paradigma a una ciencia experimental. El laboratorio se constituye en un espacio ideal ya que integra la teoría, la práctica y la experiencia de un estudiante en un único ambiente, en donde no solo se promueve el aprendizaje con el objetivo de poner en práctica la teoría vista en la clase magistral, sino también, se pueden generar nuevos conocimientos. Algunos estudios como Casotti, Rieser, Knabb (2008), Soltis, Verlinden, Kruger, Carroll y Trumbo (2015) y Moog, Creegan, Hanson, Spencer y Straumanis (2006) implementaron metodologías basadas en la exploración que evitan el método de enseñanza tradicional de transmisión de conocimiento en un solo sentido (profesor a estudiante). Para evaluar si estas implementaciones son exitosas se usan métodos cuantitativos para establecer efectos y correspondencias entre los adquiridos y las calificaciones mediante herramientas numéricas como encuestas y test, pero, ¿Cómo extraer la experiencia de los estudiantes?
Existen diversos tipos de laboratorio en Fisiología; por ejemplo, virtuales (Swift, 2016), basados en Software de simulación (Richard et al., 1994) o de experimentación en animales vivos (Shore et al., 2013). Cada tipo de laboratorio busca mejorar el proceso de enseñanza en los estudiantes basando su éxito en resultados como pruebas de conocimiento; sin embargo, se ha explorado poco sobre lo que sienten o experimentan los estudiantes frente a este tipo de experiencias de laboratorio. De acuerdo a lo anterior, este estudio se basó en la pregunta: ¿Qué se vive, que se siente y cómo perciben los laboratorios de fisiología los estudiantes de medicina veterinaria en la Universidad Nacional de Colombia? En coherencia con esta pregunta, nuestro principal objetivo fue analizar la experiencia de los estudiantes a través de la interpretación de diarios de campo construidos durante el desarrollo de los laboratorios en fisiología en un semestre académico de la asignatura Fisiología, esto con el fin de explorar los diferentes puntos de vista sobre el laboratorio provenientes de los estudiantes y reconstruir la experiencia de los mismos de acuerdo a los sucesos vividos durante el semestre académico.

\section{Materiales y métodos}

La metodología usada en los laboratorios fue el POGIL (Process oriented guided inquiry learning) o aprendizaje guiado basado en la indagación, una estrategia didáctica centrada en el estudiante, enfocada en el constructivismo, la indagación y el trabajo colaborativo. Este último se basa en la idea de que cada estudiante tiene una función específica dentro del grupo para que al final el resultado obtenido sea la integración del trabajo de todos, además emplea la corriente del "ciclo de aprendizaje", según Eberlein 
et al. (2008) en la cual un estudiante construye su entendimiento explorando, proponiendo conceptos y aplicando los mismos en su paso por el laboratorio. Lo anterior con la intención de que los estudiantes vivieran la experiencia de la experimentación libre en grupos de trabajo colaborativos de cinco personas durante su paso por el laboratorio de fisiología, esta metodología difiere al método tradicional en donde los estudiantes desarrollan el laboratorio en base a una guía de instrucciones para llegar a un resultado único esperado por el profesor.

Tomando como base la metodología utilizada por Kolkhorst et al. (2001) para la construcción de diarios de campo, se pidió a los estudiantes de Medicina Veterinaria que al final del semestre entregaran un diario personal libre en donde comentaran su proceso de aprendizaje, experiencias personales, frustraciones y logros durante el paso por cada una de las prácticas en el laboratorio. Para poder familiarizar a los estudiantes con la escritura de un diario se realizó una sesión explicativa para presentar un fragmento del diario de Ana Frank, en donde ella relata su historia como adolescente y los dos años en que permaneció oculta de los Nazis. El propósito de esta sesión fue ilustrar a los estudiantes sobre cómo plasmar su experiencia personal incluyendo sentimientos, sensaciones y emociones durante su paso por el laboratorio de fisiología.

Los estudiantes entregaron el diario completo al final de semestre después de realizar cinco prácticas de laboratorio con la metodología POGIL. La extensión y estructura del escrito fue libre. Se escogieron cinco diarios en los cuales la escritura fuera más completa y extensa, es decir, en donde se viera el interés por relatar la mayor cantidad de sucesos presentados durante el desarrollo de los laboratorios. Se realizó un análisis narrativo de cada uno de los diarios siguiendo a Connelly y Clandinin (1995) explorando las tres dimensiones narrativas: Tiempo, espacio e interacción social, este se basó en la reconstrucción de la historia de cada estudiante durante su paso por el laboratorio de fisiología de acuerdo al contexto de sus vidas y a los diferentes personajes con los que interactuaron.

La base principal para este análisis fue la creación de acontecimientos principales en la historia de cada estudiante de acuerdo a su escrito por cada práctica de laboratorio, esto debido a que cada sesión se realizó aproximadamente cada 20 días después de terminar las bases teóricas en la clase magistral. Un ejemplo de un acontecimiento fue "MI PRIMER DIA EN EL LABORATORIO", cuya base fue el escrito en un diario de estudiante de su primera práctica, aquí se utilizaron los relatos del estudiante para crear una narración sobre su experiencia en el primer laboratorio de fisiología. Los componentes principales para construir dicha narración fueron las frases plasmadas en cada relato; por ejemplo, el estudiante escribió: "fue muy chévere el hecho de que los experimentos fueran libres porque a medida que se iba aprendiendo el tema, se hacían cosas chéveres y la práctica no se tornaba aburridora". Para ir construyendo la historia de un estudiante se exploraban los componentes nuevos que aparecían en los escritos a medida que avanzaban las prácticas posteriores, esto incluyó ejemplos como nuevas relaciones sociales o el surgimiento de conflictos, nuevas opiniones negativas o positivas sobre el laboratorio, nuevas experiencias o conocimientos adquiridos en las técnicas, aplicación de lo aprendido en la 
vida cotidiana, entre otros, al final se construía una historia que iba de principio a fin. Ya que el diario era anónimo, se le dio un seudónimo al protagonista de cada historia reconstruida (ejemplo: Diario de Juan) y se realizó una reflexión sobre el papel del laboratorio en la enseñanza de la fisiología de acuerdo a la visión de los estudiantes de medicina veterinaria de la Universidad Nacional de Colombia.

\section{Resultados y discusión}

Tomando como punto de partida los relatos sobre la experiencia de los estudiantes al llevar a cabo las prácticas de fisiología durante todo un semestre académico, este punto se desarrolló a través de preguntas centrales relacionadas con lo que se vive en los laboratorios de fisiología:

- ¿EI laboratorio es un lugar para empoderar y perpetuar el conocimiento de los temas en fisiología?

Encontramos que existe una idea preconcebida de los estudiantes hacia el papel de los laboratorios en fisiología relacionada con la meta de reforzar y aplicar los conocimientos, lo anterior por escritos antes de iniciar las prácticas como: "aspiraba que fuera muy útil para el entendimiento de los temas de la clase de fisiología y su respectivo refuerzo" (Diario de Juan) y "el laboratorio es una forma más tangible de aplicar los conceptos teóricos ya que al ponerlos en práctica son mejor entendidos" (Diario de Alejandra). Estas ideas pueden demostrar dos puntos de vista: el primero se relaciona con el concepto refuerzo, aquí ya existe el conocimiento previo adquirido y el laboratorio es un apoyo para mejorar la retención y profundización del mismo.

El segundo punto de vista se relaciona con el concepto aplicar la teoría en la práctica. En este punto de vista los conoci- mientos teóricos de fisiología adquieren un sentido y una aplicación al ser visualizados en un experimento o registro de laboratorio. Lo anterior puede demostrar que la finalidad del refuerzo y la aplicación mencionados es darle una conclusión a los temas vistos en la asignatura, esta conclusión es terminar de empoderarse del tema por medio del laboratorio, como ejemplo "al realizar los laboratorios de esta forma se logra entender el tema casi en su totalidad"(Diario de Juan). Es posible que la causa de estos puntos de vista sea lo que los estudiantes referencian en sus escritos como el "método tradicional de estudio" que se lleva a cabo en las clases magistrales, en este caso de la Universidad Nacional, un método que, según ellos, busca memorizar conceptos para después evaluarlos. Algo similar a lo anterior lo comparte Ghorbani y Ghazvini (2016), quien en su metodología utiliza las prácticas de laboratorio después de la clase magistral. Afirma que en los cursos de fisiología se espera que los estudiantes memoricen un gran número de temas, y posterior, su retención memorística y comprensión serán evaluados con una calificación numérica. Es posible que de aquí surja la preocupación de los estudiantes por empoderarse de los temas y para ellos el laboratorio es concebido como un medio para lograr este objetivo.

Al observar la forma en que los estudiantes tomaban sus registros escritos sobre 
el componente teórico de cada práctica de laboratorio, encontramos que los dibujos realizados sobre algún proceso fisiológico eran una réplica del material brindado por el profesor. En la Imagen 1 se muestra un dibujo extraído del Diario de Juan y se pone en comparación con un dibujo extraído de otro estudiante publicado por Orlf (2012) en la White Mountains Community College, la principal diferencia es la manera de plasmar la información. El dibujo de Juan refleja aspectos del modelo de libro de fisiología presentado por el profesor, mientras que el dibujo del estudiante de la White Mountains
Community College demuestra una manera más personalizada y creativa de plasmar un tema, ¿es posible que de esta manera será más fácil de entender y será retenido en la mente por un mayor tiempo? De lo anterior se puede plantear que el modo de tomar apuntes de Juan sugiere la idea de que para él, la retención memorística sea la opción más viable, no por falta de creatividad, sino por superar el momento de la evaluación, por lo cual prefiere registrar el mismo modelo brindado por el profesor en vez de crear su propio modelo personal,

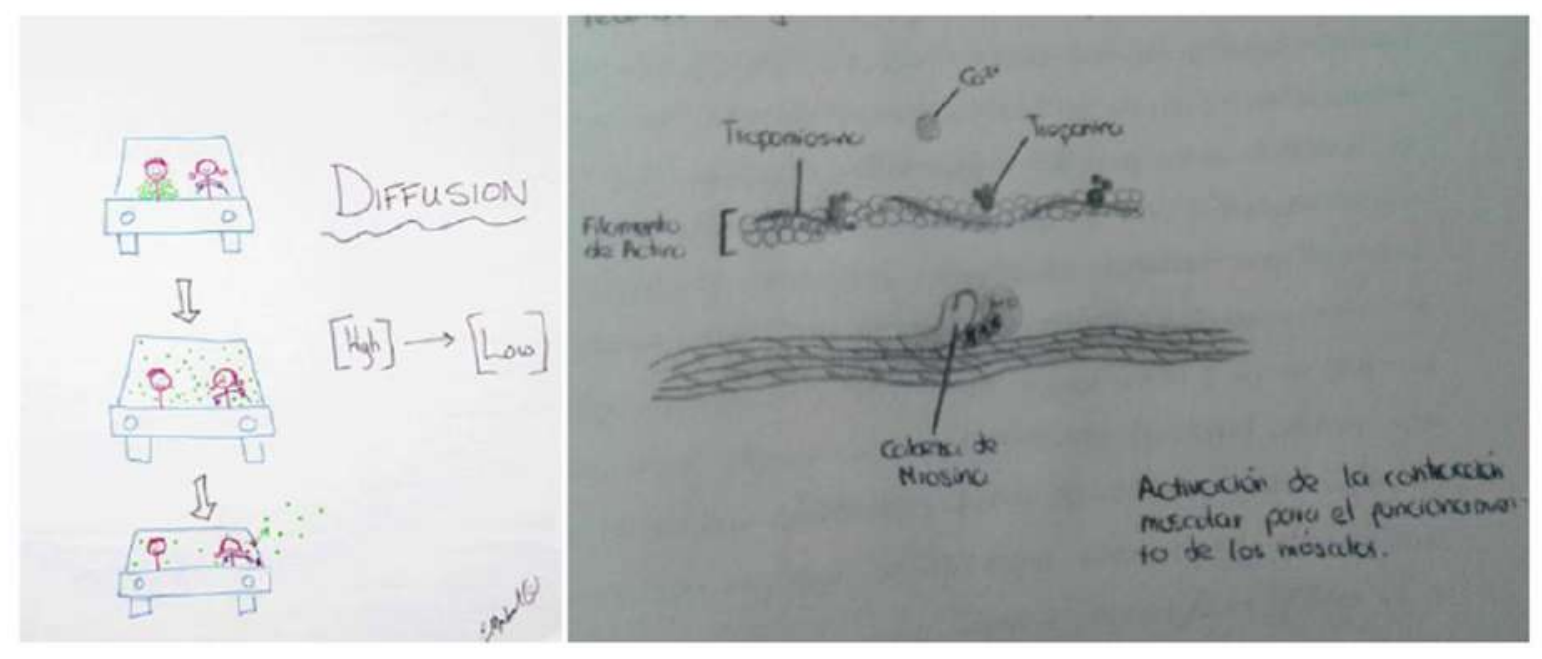

Imagen 1: es una comparativa entre el dibujo de un estudiante para recordar un proceso fisiológico extraído por la White Mountains Community College (Izquierda) y un dibujo del diario de Juan (Derecha). Imagen tomada de Orlf (2012)

Hubo una afinidad de los estudiantes hacía la base pedagógica del POGIL dirigida hacia la retención del conocimiento por medio de la formulación de hipótesis, un ejemplo de escrito: "siempre sentí que es muy bueno establecer hipótesis que se comprueben y rechacen porque a mi percepción, el conocimiento queda perpetuado a través de la conclusión sobre la experiencia y la comprobación" (Diario de Esteban). Esta afinidad es descrita por Ruiz (2013), en su obra relata que para John Dewey los estudiantes mediante la comprobación de hipótesis pueden generar experiencias tangibles que recordarán a través de los tiempos, y por Casotti et al. (2008) en donde los estudiantes afirmaron que después de las prácticas de laboratorio basadas en la indagación lograban retener los conocimientos por mucho tiempo. De lo anterior, se puede decir que el laboratorio puede ser concebido como una herramienta para perpetuar el conoci- 
miento a través del tiempo, en este caso, los estudiantes tenían la posibilidad de diseñar sus experimentos y obtener e interpretar sus propios resultados sin la necesidad de un protocolo a seguir determinado por el profesor, lo cual abre la posibilidad de juntar el conocimiento, la aplicación de la teoría y las nuevas experiencias que surjan durante el proceso de aprendizaje.

\section{- ¿Qué ocurre con la interacción social} en el laboratorio?

Los estudiantes al inicio del semestre concebían el trabajo en equipo como un medio para mejorar el entendimiento de los temas; sin embargo, con el pasar del tiempo relataron un nuevo complemento sobre el trabajo en grupo, el cual ya no solo comprendía el empoderamiento de los temas, sino el sentimiento de agrado al hecho de compartir con otras personas, "es grato poder comentar que el proceso de aprendizaje pasa de ser una responsabilidad estrictamente dicha para convertirse en un gusto, cuando se lleva a cabo en buena compañía $y$ entre risas, claramente con la seriedad que corresponde a un proceso importante pero acompañado de la humanidad que nos define ......" (Diario de Linda). Este hallazgo relacionado con el ambiente cordial en el laboratorio también es descrito por Soltis y colaboradores (Soltis et al., 2015) quienes afirman que la experimentación libre mantuvo a los estudiantes enfocados en el trabajo y les dio a entender que existen beneficios en el trabajo grupal. De lo anterior se puede decir que al permitir que los estudiantes desarrollaran sus experimentos de manera libre y autónoma en donde el trabajo colaborativo era imprescindible para el desarrollo del laboratorio, se puede crear un ambiente de convivencia, en donde naturalmente se empezaron a compartir gustos $\mathrm{y}$ opiniones similares que iban más allá de lo académico. Ellos lograron mantener su objetivo de "dominar los temas" pero añadiendo el "agrado al trabajo en el laboratorio", percibiendo que el laboratorio no solo es un lugar académico sino también un espacio para compartir ideas personales, un lugar en donde se puede estudiar, adquirir conocimiento y generar empatía al mismo tiempo reforzando la relación colaborativa que será de utilidad en la formación de futuros colegas profesionales.

No todos los aspectos del trabajo en grupo fueron positivos, al analizar los diarios escritos encontramos sensaciones negativas como inseguridad, dificultad para hacer consensos entre estudiantes, inconvenientes para la obtención de registros, incompatibilidad de personas con historial de malas experiencias, dificultad en el desempeño de trabajo y la expulsión de integrantes de un grupo. Por ejemplo, Alejandra escribió:., "me siento insegura porque los grupos son grandes y es dificil poner de acuerdo a tanta gente, espero no tener inconvenientes"; "habían muchas perturbaciones en el medio como compañeros hablando y riéndose aumentando la actividad cerebral (Diario de Alejandra)"; "nos hicimos con algunos amigos y otros no tanto" (Diario de Cristina) y "para la presentación de la guía mis compañeras amablemente me sacaron del grupo, según ellas, no había ayudado suficiente" (Diario de Cristina). Estas experiencias pueden sugerir indicios de problemas con el trabajo en grupo en otras asignaturas pasadas, también puede ser por la afinidad natural o costumbre de algunos estudiantes al trabajo individual y no al grupal, o tal vez la presión de obtener resultados pensando en la futura evaluación y la calificación genera estos inconvenientes. Adicional a lo anterior, Soltis et al. (2015), 
al estudiar la convivencia de los grupos de trabajo en el laboratorio, describieron causas negativas similares como la no asistencia a clase, estar desprevenido, o no tener el deseo de participar en los ejercicios. De estos hallazgos se puede inferir que uno de los factores que puede convertir al laboratorio en una experiencia poco agradable puede ser la interacción de los estudiantes y el tiempo compartido en el grupo de trabajo.

$\mathrm{Al}$ inicio de la primera práctica, al escuchar varios comentarios de los estudiantes dentro de clase, la figura docente se relacionaba con el concepto de "autoridad" o "sabiduría sin derecho a contradicción por parte del estudiante" o "tiene la última palabra". De acuerdo a la metodología implementada en los laboratorios, se buscaba que el profesor no representara una figura de autoridad o un transmisor de información en un solo sentido, se esperaba que fuera un facilitador del aprendizaje al brindar los mejores recursos y arreglar el entorno en el que interactuarían los grupos de trabajo, con esto los estudiantes, despertaron confianza para desarrollar sus experimentos sin ningún tipo de exigencia, un ejemplo de escrito: "Es muy satisfactorio que el docente deposite su confianza en el estudiante asumiendo que con su creatividad e intriga sea capaz de entender el tema por sí solo" (Diario de Juan). Este tipo de relación genera una motivación en los estudiantes que puede llegar a mejorar su capacidad para desarrollar habilidades sociales, emocionales y cognitivas en cualquier entorno de la vida académica.

Al analizar los momentos en donde se debían exponer los resultados obtenidos frente a los compañeros, fue común encontrar sentimientos de temor e incomodidad, “algunos grupos pasaron a explicar, mientras tanto mi grupo y yo discutíamos acerca de quién sería la persona encargada de pasar ya que algunos estaban nerviosos" (Diario de Juan); "como cualquier socialización estaba muerta del miedo" (Diario de Alejandra) y "me produce mucha ansiedad tener que pasar al frente a exponer y la presencia de un profesor aumenta la presión" (Diario de Alejandra). Estudios como Casotti et al. (2008) y Hanson (2006) afirman que los estudiantes después de realizar los laboratorios con experimentación libre aumentaron su confianza al presentar públicamente sus resultados, lo cual no se observó en los estudiantes de veterinaria que participaron en este estudio. Estos relatos encontrados demuestran sentimientos negativos a pesar de que no existían resultados buenos o malos al momento de socializar en público, ya que la idea era fomentar una discusión constructiva a partir de los mismos. Es posible que lo anterior se deba a que los estudiantes en su rutina educativa esperan que cualquier evento para demostrar su trabajo va a ser susceptible de una calificación o una crítica, también, la propia personalidad reservada de algunos estudiantes o diferentes experiencias negativas en el pasado al momento de presentar ideas en público pudieron contribuir, dando como resultado que el ambiente en estos espacios no reflejara confianza sino ansiedad por superar una prueba. Es posible que con el tiempo y con un número mayor de sesiones de socialización, los estudiantes puedan adquirir la confianza necesaria para presentar sus trabajos dejando los sentimientos negativos a un lado.

\section{- ¿Existe moralidad y bienestar animal en el laboratorio?}

Uno de los laboratorios sobre conducción nerviosa requería el sacrificio de una lombriz de tierra que los estudiantes debían conseguir para obtener el registro. El análisis 
de la visión de los estudiantes hacia los animales como objeto de estudio permitió encontrar en ellos sentimientos de compasión; por ejemplo: "a pesar de ser tan pequeño e inofensivo, es un ser sintiente..." (Diario de Juan), “una pequeña lombriz que generaba compasión, curiosidad, estupor o simplemente gracia” (Diario de Linda). Richard y sus colaboradores (Richard et al., 1994), en su estudio, encontraron que por razones emocionales o morales los estudiantes se molestaban por el uso de animales vivos en el laboratorio, esta razón también se encontró en nuestro estudio, "no fui capaz de atravesarla con los alfileres, no por asco, sino porque me produce malestar maltratar un ser vivo" (Diario de Alejandra).

Las razones por las cuales se puede llegar a despertar este tipo de sentimientos en los estudiantes pueden ser diversas. En este estudio, al igual que en Richard et al. (1994), una importante causa de este sentimiento proviene de la ideología del movimiento del bienestar animal, el cual ha tenido un gran impacto sobre los estudiantes en los últimos años por su presencia en los medios de comunicación y su inclusión en los programas de estudio de medicina veterinaria. Este movimiento aleja cada vez más la iniciativa de la experimentación con animales vivos. Otra causa posible es la empatía natural que tienen los seres humanos hacía los seres que consideramos inferiores acompañado de un sentimiento de culpa al usar un ser vivo para empoderarse del conocimiento, "tratando de tener el mayor cuidado posible con el animal aun a expensas de saber que inevitablemente iba a morir" (Diario de Linda).

A pesar de que existe una cátedra de bienestar animal en el plan de estudios de
Medicina Veterinaria en la Universidad Nacional de Colombia, y esta intenta concientizar a los estudiantes sobre la reducción y el reemplazo del uso de animales vivos en prácticas experimentales, al momento de analizar qué tan necesario es el uso de estos en los laboratorios de fisiología, encontramos que los estudiantes de veterinaria acuden al concepto aprendizaje, un ejemplo de escrito: "saber que iba a morir no era un sentimiento grato, de igual manera, todo sea en nombre del aprendizaje" (Diario de Linda) y "sé que este tipo de situaciones son necesarias para el aprendizaje y trato de aprovecharlas igualmente" (Diario de Alejandra). Un hallazgo similar es descrito por Beatriz et al. (2016), en su estudio, los estudiantes experimentaron molestias con el uso de animales en el laboratorio, pero creen que es importante para su aprendizaje, incluso los estudiantes que no se sentían cómodos en las prácticas todavía participaron en estas actividades. Estos hechos demuestran que existe un conflicto moral en el uso de animales para la enseñanza de la fisiología; sin embargo, cuando se presenta la oportunidad de obtener conocimiento, este sentido moral se desplaza, posiblemente porque los estudiantes al experimentar con el animal pueden poner en práctica la teoría la cual conduce de nuevo, al empoderamiento de los temas. ¿Es necesario el uso de animales para la enseñanza de la fisiología?, como se mencionó anteriormente, existen laboratorios virtuales o de simulación que podrían aportar los mismos conocimientos extraídos de una práctica con animales vivos; sin embargo, como se visualizó en el relato anterior o como lo comparte Alice (2005) la forma más efectiva de ayudar a los estudiantes a entender las interacciones fisiológicas se logran con una experiencia en un modelo vivo, no obstante, queda en duda de si la causa por la cual los estudiantes continúan con estos experimen- 
tos a pesar de sentir molestia e incomodidad es la evaluación y calificación, esto debido a que los equipos del laboratorio solo están configurados para la práctica con la lombriz y ningún estudiante se opuso o propuso una alternativa que no involucrara el sacrificio del animal para el desarrollo de la sesión.

\section{- ¿EI laboratorio puede ser un espacio} de agrado fuera de la rutina académica?

Hasta el momento encontramos que el laboratorio tiene espacio para el empoderamiento de los temas, la interacción social y la moralidad, ¿podría convertirse en un sitio de agrado y diferente?, los estudiantes de veterinaria podrían dar una respuesta positiva a esta pregunta, "trabajamos mientras teníamos la oportunidad de reír y tomarnos con gracia la seriedad de los conceptos trabajando activamente" (Diario de Linda), "la dinámica del laboratorio nos oxigenaba de la densidad de la cotidianidad académica que en ocasiones se torna monótona, cuadriculada y pesada" (Diario de Linda). Otro hallazgo muy importante se refleja en el relato: "me gusta mucho descubrir cosas en el laboratorio utilizando el modelo que sea, me ayuda a salir de la monotonía" (Diario de Esteban). Como se mencionó anteriormente, el laboratorio al inicio de las prácticas era concebido como un lugar académico-evaluativo que, posiblemente en la visión inicial de los estudiantes, no iba a tener nada diferente a lo vivido en otras asignaturas; sin embargo, con el pasar del tiempo se evidencia una sensación de motivación en donde el laboratorio se convierte en un lugar diferente, un lugar que los estudiantes describen como de "oxigenación" de la rutina académica, como si en todas las asignaturas se llevara a cabo metodologías similares que nunca cambian y que se describen como "aburridas y repetitivas", posiblemente por la carga de clases magistrales que se llevan a diario en la academia. Es posible que estas emociones surjan en los estudiantes por el hecho de que no existía ninguna evaluación o calificación en el desarrollo de los laboratorios y, sumado a un ambiente de cordialidad, puede llegar a disminuir la presión de obtener resultados académicos dándole paso a la curiosidad y al agrado

- ¿El laboratorio aplicado a la vida le da un sentido?

Desde el inicio de los laboratorios fue evidente el impulso de los estudiantes por extrapolar lo que veían en los experimentos a situaciones de la vida diaria, por ejemplo, al hablar sobre medios tónicos un estudiante se preguntó: "como se comportarían mis vasos si les agrego algo concentrado en el líquido extracelular" (Diario de Esteban). Otro ejemplo ocurrió al realizar una práctica de electroencefalografía, en donde se evaluaba la actividad mental con diferentes géneros musicales y volúmenes a diferentes niveles de intensidad, de aquí surgió la idea: "concluyo que la música a alto volumen implica una actividad de no descanso o relajación para mi cerebro, por lo cual, me doy cuenta que cuando vaya a estudiar es preferible escuchar música a bajo volumen y no alto" (Diario de Esteban). De acuerdo a Ahrash y Lemons (2006) la taxonomía de Bloom afirma que los estudiantes después de un curso teórico práctico pueden desarrollar habilidades de aprendizaje que no requieren el pensamiento crítico, estas son el conocimiento básico (memorizar los conceptos) y comprensión secundaria (entender los conceptos), y como lo afirma Ahrash y Lemons (2006), solo la habilidad de la aplicación 
(relacionar los conceptos con otro ámbito de la vida) requiere pensamiento crítico. Este hallazgo puede ser compatible con Soltis et al. (2015) quienes afirman que hay un mayor rendimiento en el pensamiento de orden superior como la aplicación y el análisis en los estudiantes después vivir experiencias en el laboratorio. Debido a que la metodología del laboratorio solo aportaba medios para adquirir conocimiento en fisiología sin ninguna extrapolación práctica, los relatos anteriores sugieren que, aunque no implícita, pudo estar presente la aplicación ya que los estudiantes de manera natural en sus relatos no describían los conocimientos adquiridos en el laboratorio sino intentaron generalizar los conceptos aprendidos en contextos diferentes de la vida cotidiana. Es posible que todos los conocimientos adquiridos en el laboratorio tengan un sentido o una razón de ser en el momento en el que se aplica en una situación conocida, en este caso para ellos, tuvo sentido en la vida cotidiana de un estudiante común.

En los escritos de varios estudiantes eran evidentes emociones que surgían a medida que transcurrían las prácticas de laboratorio, estas eran dirigidas hacía la palabra vida y como el laboratorio les ayudaba a explorarla, "esta es la práctica que más me ha gustado y la que más me hace sentir la sensación de vida.... Utilizar un fonendoscopio y escuchar que un organismo está trabajando es genial" (Diario de Esteban). Es posible que estas percepciones surgieron en los estudiantes al vivir experiencias en el laboratorio de fisiología al tener contacto directo práctico, esto debido a que la mayoría de temas vistos en Fisiología se llevan a cabo de manera teórica en la clase magistral. Otro hallazgo importante es el papel que puede tomar el laboratorio cuando los estudiantes lo relacionan con la vida y no con lo académico, "los laboratorios en fisiología son importantes para el estudiante ya que permiten evidenciar la vida y sus mecanismos de funcionamiento y no solo la dinámica de aprobar o reprobar una materia con parciales" (Diario de Esteban), de lo anterior se puede decir que la visión inicial del laboratorio como una herramienta para empoderar el conocimiento se complementa con la curiosidad y el entusiasmo por explorar la naturaleza del organismo. Esto puede generar ventajas en la enseñanza y aprendizaje de la Fisiología ya que el estudiante tendrá una motivación adicional aparte de la evaluación y la calificación para el estudio del organismo, que quizás se desarrolle en un gusto específico que pueda ser una guía para el estudiante en su futuro académico.

\section{Conclusión}

口-

Mientras que muchos estudios evalúan la efectividad de la implementación de nuevas didácticas y pedagogías en el laboratorio de fisiología por medio de resultados cuantitativos como encuestas, promedios y calificaciones, este estudio exploró aspectos cualitativos como la visión de los estudiantes frente al laboratorio y su experiencia vivida al realizar las prácticas durante el semestre académico. Al responder a la pregunta: ¿Qué se vive, que se siente y cómo perciben los laboratorios de fisiología los estudiantes de medicina veterinaria en la Universidad Nacional de Colombia? Se encontraron 
diferentes aspectos gracias al uso de la narrativa. Uno de ellos es que los estudiantes definen al laboratorio como una herramienta para reforzar, aplicar y perpetuar el conocimiento en fisiología que al parecer es medio útil para satisfacer la preocupación del empoderamiento de los temas. Al hablar de aspectos sociales y personales se evidenciaron varios factores que pueden llevar a experiencias negativas en el laboratorio, uno de ellos es la convivencia de las personas y los conflictos que se presentaron al trabajar en grupo; sin embargo, hubo casos en donde se evidenció agrado al poder estudiar y generar empatía al mismo tiempo, aquí el laboratorio se concebía como un lugar en donde los estudiantes podían escapar por un momento de la monotonía en la rutina académica. Adicional a lo anterior, también se encontró un conflicto moral en los estudiantes al utilizar animales vivos en los experimentos de fisiología ya que, al presentarse la oportunidad de obtener conocimiento, todo sentimiento de compasión y empatía queda a un lado debido a la necesidad del aprendizaje.

El uso de la investigación narrativa nos permitió explorar a profundidad una mayor cantidad de elementos que podrían estar ocultos al utilizar solamente los métodos cuantitativos, con hechos como por ejemplo que el laboratorio puede ser un lugar que genera curiosidad y entusiasmo por explorar la naturaleza del organismo. Todos estos aspectos pueden servir de base y ayuda para guiar a los maestros de Fisiología que intentan explorar nuevos caminos de innovación para mejorar el proceso de enseñanza y aprendizaje de la fisiología experimental, con lo cual se podrán tener en cuenta estos y muchos más elementos nuevos para la implementación de nuevas didácticas en el laboratorio.

\section{REFERENCIAS BIBLIOGRÁFICAS}

Ahrash, N. y Lemons, P. (2006). A New Method For Assessing Critical Thinking In The Classroom, Bioscience, 56(1), 66 - 77

Alice, W. (2005). The Evolving Role Of Animal Laboratories In Physiology Instruction, Adv Physiol Educ, 29, 144-150

Beatriz, F. Rochelle, R. Sonia, R. Ricardo, H. Silva, A. Maria, J. Rocha, R. (2016), Perceptions Of Undergraduate Students On The Use Of Animals In Practical Clases, Adv Physiol Educ 40, PP. $422-424$

Casotti, G.; Rieser, D. y Knabb, M. (2008), Successful Implementation Of Inquiry-Based Physiology Laboratories In Undergraduate Major And Nonmajor Courses, Adv Physiol Educ, 32, 286-296

Connelly, M. y Clandinin, J. (1995). Relatos De Experiencia E Investigación Narrativa: Hoy Todos Son Constructivistas, Cuadernos De Pedagogía, 257, 78-84

Eberlein, T. Jack, K. Vicky, M. Moog, R. Terry, P. Pratibha, V. White, H. (2008), Pedagogies of engagement in science: A comparison of PBL, POGIL, and PLTL, Biochemistry And Molecular Biology Education 36(4), 262-273, 
Ghorbani, A. Ghazvini, K. (2016), Using Paper Presentation Breaks During Didactic Lectures Improves Learning Of Physiology In Undergraduate Students, Adv Physiol Educ 40, 93-97

Hanson, D. (2e006). Instructor's Guide to Process-Oriented Guided-Inquiry Learning, Department of Chemistry, Stony Brook University, New York: Pacific Crest,

Kolkhorst, F.; Mason, C.; Dana, M.; Patricia, P. y Michael, J. (2001). An Inquiry-Based Learning Model For An Exercise Physiology Laboratory, Adv Physiol Educ, 25, 45-50

Moog, R.; Creegan, F.; Hanson, D.; Spencer, J. y Straumanis, A (2006), Process-Oriented Guided Inquiry Learning: Pogil And The Pogil Project, Metropol Univ J, 17, 41-51

Orlf, M. (2012). Real-Time Drawings: Sketches That Help Students Understand Difficult Concepts, Has Educator, 16(2), 25-27

Richard, W.; Gregory, A.; Schmidt, J.; Lawrence, D.; Sanjeev, G. y Paul, T. (1994), Cardiovascular Physiology Teaching: Computer Simulations Vs. Animal Demonstrations, Adv. Physiol. Educ, 266, S.6-S.46

Ruiz, G. (2013). La Teoría De La Experiencia De John Dewey: Significación Histórica Y Vigencia En El Debate Teórico Contemporáneo, Foro De Educación, 11(15), 103-124

Shore, N.; Shireen, K.; Miraa, Q. y Muhammad, A. (2013), Animal Laboratory, Interactive And Computer Based Learning In Enhancing Basic Concepts In Physiology: An Outlook Of 481 Undergraduate Medical Students, Ayub Med Coll Abbottabad, 25(1-2), 57-59

Soltis, R.; Verlinden, N.; Kruger, N.; Carroll, A. y Trumbo, T. (2015), Process-Oriented Guided Inquiry Learning Strategy Enhances Students' Higher Level Thinking Skills In A Pharmaceutical Sciences Course, American Journal Of Pharmaceutical Education, 79(1), 1-8

Swift, A. (2016). Is Labtutor A Helpful Component Of The Blended Learning Approach To Biosciences? Journal Of Clinical Nursing, 25 (17-18), 2683-2693 